\title{
Quantum Optics with Single Nanowire Quantum Dots
}

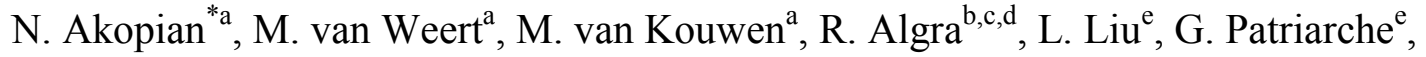 \\ J.-C. Harmand ${ }^{\mathrm{e}}$, E. Bakkers ${ }^{\mathrm{c}}$, L. Kouwenhoven ${ }^{\mathrm{a}}$, V. Zwiller ${ }^{\mathrm{a}}$ \\ ${ }^{a}$ Kavli Institute of Nanoscience, Delft University of Technology, 2628CJ Delft, The Netherlands; \\ ${ }^{b}$ Materials Innovation Institute (M2i), 2628CD Delft, The Netherlands; \\ 'Philips Research Laboratories Eindhoven, High Tech Campus 11, 5656AE Eindhoven, The \\ Netherlands; \\ d3IMM, Solid State Chemistry, Radboud University Nzjmegen, Heijendaalseweg 135, 6525AJ \\ Nijmegen, The Netherlands; \\ ${ }^{\mathrm{e}}$ Laboratoire de Photonique et de Nanostructures, CNRS, route de Nozay, 91460 Marcoussis, France
}

\begin{abstract}
In this paper we present our recent developments in control and manipulation of individual spins and photons in a single nanowire quantum dot. Specific examples include demonstration of optical excitation of single spin states, charge tunable quantum devices and single photon sources. We will also discuss our recent discovery of a new type of charge confinement - crystal phase quantum dots. They are formed from the same material with different crystal structure, and today can only be realized in nanowires.
\end{abstract}

Keywords: nanowires, quantum dots, crystal phase

\section{INTRODUCTION}

Nanowires are defined as structures that have a lateral size constrained to tens of nanometers or less and an unconstrained longitudinal length. At these scales, quantum mechanical effects become important, therefore, such wires are also known as "quantum wires". Many different types of nanowires exist today, including metallic (e.g., Ni, Pt, Ag, $\mathrm{Au}$ ), semiconducting (e.g., $\mathrm{Si}^{2}, \mathrm{InP}^{3}, \mathrm{InAs}^{4}, \mathrm{GaAs}^{5,6}, \mathrm{GaN}^{7}$ ), and dielectrics (e.g., $\mathrm{SiO}_{2}, \mathrm{TiO}_{2}$ ). Typical nanowires exhibit aspect ratios (length-to-width ratio) of 1000 or more.

The nanowires we study could be used to link or to address components into extremely small circuits. There are many applications where nanowires may become important in electronics, opto-electronics and nano-electromechanical devices, as additives in advanced composites, for metallic interconnects in nanoscale quantum devices, as field-emitters and as biomolecular nanosensors.

The nanowires we study are fabricated "bottom-up" using self-assembly in contrast to techniques based on etching. To create active photonic elements, the first key step is to incorporate a single quantum dot in a nanowire ${ }^{8}$. Semiconductor quantum dots are well known sources of single $e^{9-11}$ and entangled photons ${ }^{12-14}$ and are naturally integrated with modern semiconductor electronics. Incorporation in semiconducting nanowires brings additional unique features such as natural alignment of vertically stacked quantum dots to design quantum dot molecules and an inherent one-dimensional channel for charge carriers. Furthermore, the unprecedented material and design freedom makes them very attractive for novel opto-electronic devices and quantum information processing.

In this paper we present our recent progress in understanding the physical properties of single nanowire quantum dots, nanowire based devices and our recent discovery - crystal phase quantum dots in nanowires. We first describe two growth techniques that are used to grow optically active nanowire quantum dots of good quality with narrow emission lines, enabling us to access the spin properties of the exciton and we show a charge tunable device based on a single nanowire quantum dot. We also demonstrate generation of single photons from crystal phase quantum dots.

*n.akopian@tudelft.nl

Quantum Sensing and Nanophotonic Devices VII, edited by Manijeh Razeghi, Rengarajan Sudharsanan, Gail J. Brown, Proc. of SPIE Vol. 7608, 76080T - (C) 2010 SPIE · CCC code: 0277-786X/10/\$18 - doi: 10.1117/12.840481 


\section{NANOWIRE QUANTUM DOTS}

\subsection{Growth}

Nanowires can be fabricated in numerous ways. Metallic nanowires are usually synthesized in suspension. Silicon nanowires can be etched from a silicon-on-insulator wafer, using conventional complementary metal-oxidesemiconductor (CMOS)-compatible techniques. Contrary to this top-down approach, a bottom-up approach based on the Vapor-Liquid-Solid (VLS) synthesis method may be used to grow semiconductor nanowires. The VLS method can be implemented with several growth techniques such as vapor phase epitaxy, laser ablation, metal-organic vapor phase epitaxy (MOVPE), chemical beam epitaxy, and molecular beam epitaxy (MBE). Nanowires of Si/Ge, III-V or II-VI compounds have been fabricated. The process starts with the deposition of metallic particles, usually nanometer sized gold particles, on the substrate before growth. These nano-particles can act as chemical or physical catalysts. At growth temperature, they generally form liquid droplets with the semiconductor constituents. When the sample is exposed to a supersaturated vapor flow of source materials, the concentration of these constituents in the liquid phase increases until they condensate at the droplet/substrate interface: the nanowires grow. The chemical catalyst effect consists in the preferential decomposition of precursor materials, such as organo-metallics used in MOVPE, at the surface of the metal droplets. The growth rate is then locally enhanced because free constituents are more abundant near the droplets. This is the dominant mechanism for nanowire growth by MOVPE. However, this effect does not operate in MBE where elemental constituents are supplied to the sample. In that case, the faster nucleation rate at the droplet/substrate interface results from a physical catalytic effect: the periphery of the droplet, boundary between the 3 phases, vapor, liquid and solid, presents a lower energy barrier to the nucleation. This effect also leads to nanowire formation. In both cases, the final nanowire length can be adjusted by controlling the growth time, while the diameter is set by the catalyst particle size.

Nanowire geometry offers further original possibilities to fabricate heterostructures: growth conditions can be varied in order to switch from axial growth, promoted by the catalytic effects explained above, to radial growth where material nucleates on the nanowire sidewalls. This process results in core-shell heterostructures of particular interest to shield an active core region by a high bandgap shell material.

An additional advantage of nanowires over conventional two-dimensional growth is the flexibility in structuring highly lattice-mismatched material systems: because of the very small diameter, strain can relax along the nanowire sidewalls without introducing dislocations at the mismatched interfaces, increasing the choice of material combinations as compared to two dimensional growth. This advantage may however be limited in radial heretostructures, where the core can be strained by the shell. The crystal structure in nanowires can be zincblende as for usual bulk III-V materials, but very often the wurtzite structure is found to dominate. This could be seen as another advantageous flexibility over two dimensional growth, at least when the perfect control of this crystalline phase via growth parameters will be demonstrated.

In the following we describe the fabrication of nanowires by two different techniques: MOVPE and MBE growth performed with gold nanoparticles.

\section{Metal organic vapor phase epitaxy with gold catalysts}

For some of our experiments we used single $\operatorname{InAs} \mathrm{s}_{0.25} \mathrm{P}_{0.75}$ quantum dots embedded in InP nanowires, grown by metalorganic vapor-phase epitaxy. Colloidal gold particles of $20 \mathrm{~nm}$ diameter were deposited on a (111)B InP substrate as catalysts for vertical nanowire growth. The nanowires were grown at a temperature of $420^{\circ} \mathrm{C}$, using phosphine $\left(\mathrm{PH}_{3}\right)$ and trimethylindium (TMI) as precursors. After 10 minutes of InP growth, an InAsP section was grown by introducing arsine $\left(\mathrm{AsH}_{3}\right)$ into the reactor. The fraction of As in the InAsP section was controlled by the ratio between the molar fractions of $\mathrm{PH}_{3}$ and $\mathrm{AsH}_{3}$. After 2 seconds of InAsP growth, the $\mathrm{AsH}_{3}$ flow was stopped and 10 minutes of InP growth was continued. Subsequently a thin InP shell of $40 \mathrm{~nm}$ was grown around the quantum dot by raising the temperature to $600^{\circ} \mathrm{C}$. This shell passivates the surface of the quantum dot. The diameters of the nanowire and the quantum dot were controlled by the gold particle size, while the nanowire density was set by the gold particle density on the substrate. The quantum dot height and nanowire length were determined by growth time. By controlling diameter, height and As concentration one can tune the quantum dot emission over a wide range from $900 \mathrm{~nm}$ to $1.6 \mu \mathrm{m}$. Under the growth conditions described previously we were able to grow a sample with low density of nanowires containing a single quantum dot that emits around $950 \mathrm{~nm}$. In Fig. 1 we show a high angle annular dark field transmission electron microscope (HAADF TEM) image of a nanowire quantum dot grown by MOVPE by the Bakkers group. The quantum dot is $9 \mathrm{~nm}$ high with a diameter of $31 \mathrm{~nm}$, determined by energy dispersive X-ray analysis in a TEM. Both nanowire and 
quantum dot have a wurtzite crystal structure. To simplify TEM studies, the InP section following quantum dot growth was kept short, while for the samples used in our experiments, the quantum dot was centered in the nanowire.

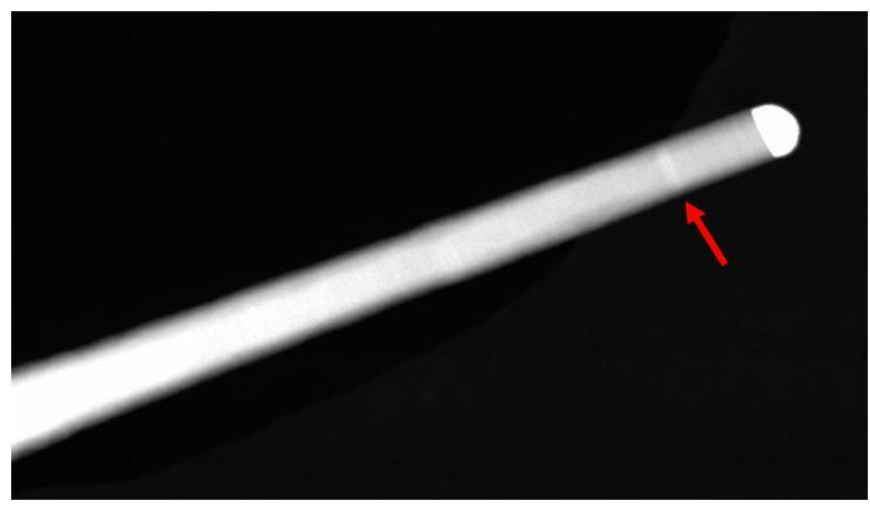

Figure 1. TEM image of an InP nanowire with an InAsP section. The gold catalyst is visible on top of the wire, the arrow points at the InAsP segment which has a width of $31 \mathrm{~nm}$. MOVPE grown samples by the Bakkers group.

\section{Molecular beam epitaxy}

Growth by Molecular Beam Epitaxy has been performed by the Harmand group. InP nanowires were fabricated by a bottom-up approach based on the Vapor-Liquid-Solid synthesis method implemented with molecular beam epitaxy growth technique on (111) B oriented InP substrates using $\mathrm{Au}$ as a catalyst ${ }^{15,16}$. The growth temperature is one of the parameters which influence the crystal phase of the nanowire. A low growth temperature $\left(380^{\circ} \mathrm{C}\right)$ is favorable to pure wurtzite InP while the formation of zinc-blende InP sections are highly probable at higher temperature $\left(420{ }^{\circ} \mathrm{C}\right)$. The samples under study were grown with two growth steps at high and low temperatures, successively.

\subsection{Optical properties}

We study the optical properties of samples grown by the techniques described above. We concentrate on InP nanowires including InAsP segments with heights shorter than $10 \mathrm{~nm}$ to produce systems with a quantum dot like behavior. The emission linewidth at $4.2 \mathrm{~K}$ is close to our spectrometer resolution $(30 \mu \mathrm{eV})$. The excitation power dependence reveals a clear exciton-biexciton behavior.

In Fig. 2a we show a typical excitation power dependence of photoluminescence (PL) revealing a usual excitonbiexciton behavior and a p-shell at $30 \mathrm{meV}$ higher energy. The inset shows the narrowest emission we have observed to date with a FWHM of $31 \mu \mathrm{eV}$, limited by our spectral resolution. The integrated photoluminescence intensities of the exciton and biexciton as a function of excitation power, represented in fig. $2 \mathrm{~b}$, show that the exciton (biexciton) increases linearly (quadratically) with excitation power and saturates at high excitation powers. This behavior is typical for the exciton and biexciton under continuous wave laser excitation. 


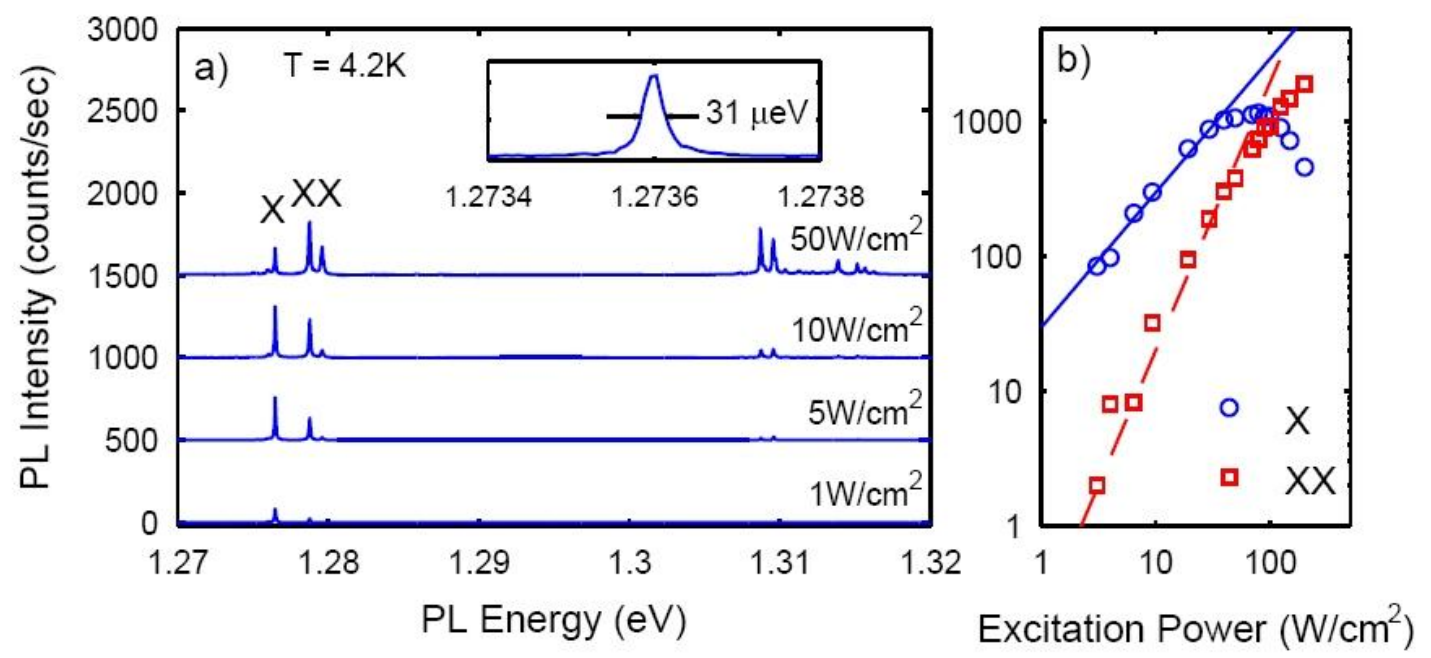

Figure 2. (a) Power dependence of the PL from a single quantum dot in a nanowire under continuous wave laser excitation at $532 \mathrm{~nm}$. (b) Integrated power dependence of two narrow emission lines attributed to the exciton and the biexciton. Samples grown by the Bakkers group.

\subsection{Optical excitation of single spin states}

A specific spin state can be excited when using either $\sigma^{+}$or $\sigma$ - polarized light. If the emitted photons have the same polarization, it means that no spin relaxation has occurred during this excitation-relaxation cycle. Although this cycle is short (on the order of a radiative lifetime of about $1 \mathrm{~ns}$ ) it represents a first step toward an exciton-spin memory. A complete spin memory also requires the ability to suppress the radiative decay for a controllable storage time, as has been demonstrated with self-assembled dots ${ }^{17}$. We have found that excitation above the InP band gap with subsequent relaxation into the dot scrambles up the spin state and thus destroys any spin memory effect. The phase space for relaxation is largely reduced when we excite below the InP band gap into one of the confined higher energy states of the dot. This relaxation between confined states is spin conserving, as we now demonstrate.

We characterize the higher energy states using combined PL and PLE (photoluminescence excitation) and identify a pshell resonance around $1300 \mathrm{meV}$ (see Fig.2 a). We use quasi-resonant excitation into this p-shell and measure the exciton luminescence for various polarization analyzers. Fig. 3 a) is taken with linear excitation and analyzer such that both spin states are excited and measured. As a function of magnetic field, the exciton transition shows a Zeeman splitting and a diamagnetic shift. Panels b) and c) in Fig. 3 show the results for left and right circularly polarized excitation. When exciting with right circular polarization, only the spin up branch of the Zeeman split exciton is populated. In this case, we dominantly observe luminescence from the high-energy peak, which is also right-circularly polarized. We thus clearly observe spin memory ${ }^{18}$. Close inspection reveals that the spin memory is not perfect and that a small peak for the wrong polarization is also observed. For nonzero $\mathrm{B}$, the peak-height ratio is $\sim 10$. However, at $\mathrm{B}=0$, a reduced polarization ratio $\sim 3$ was found. This likely indicates spin relaxation mediated by the hyperfine interaction with nuclear spins ${ }^{19}$. Similar measurements have been reported on ensembles of self-assembled quantum dots under quasiresonant ${ }^{20}$ and resonant excitation ${ }^{21}$. However, polarization memory was not observed at $\mathrm{B}=0$, possibly because of a larger exciton fine structure splitting. 

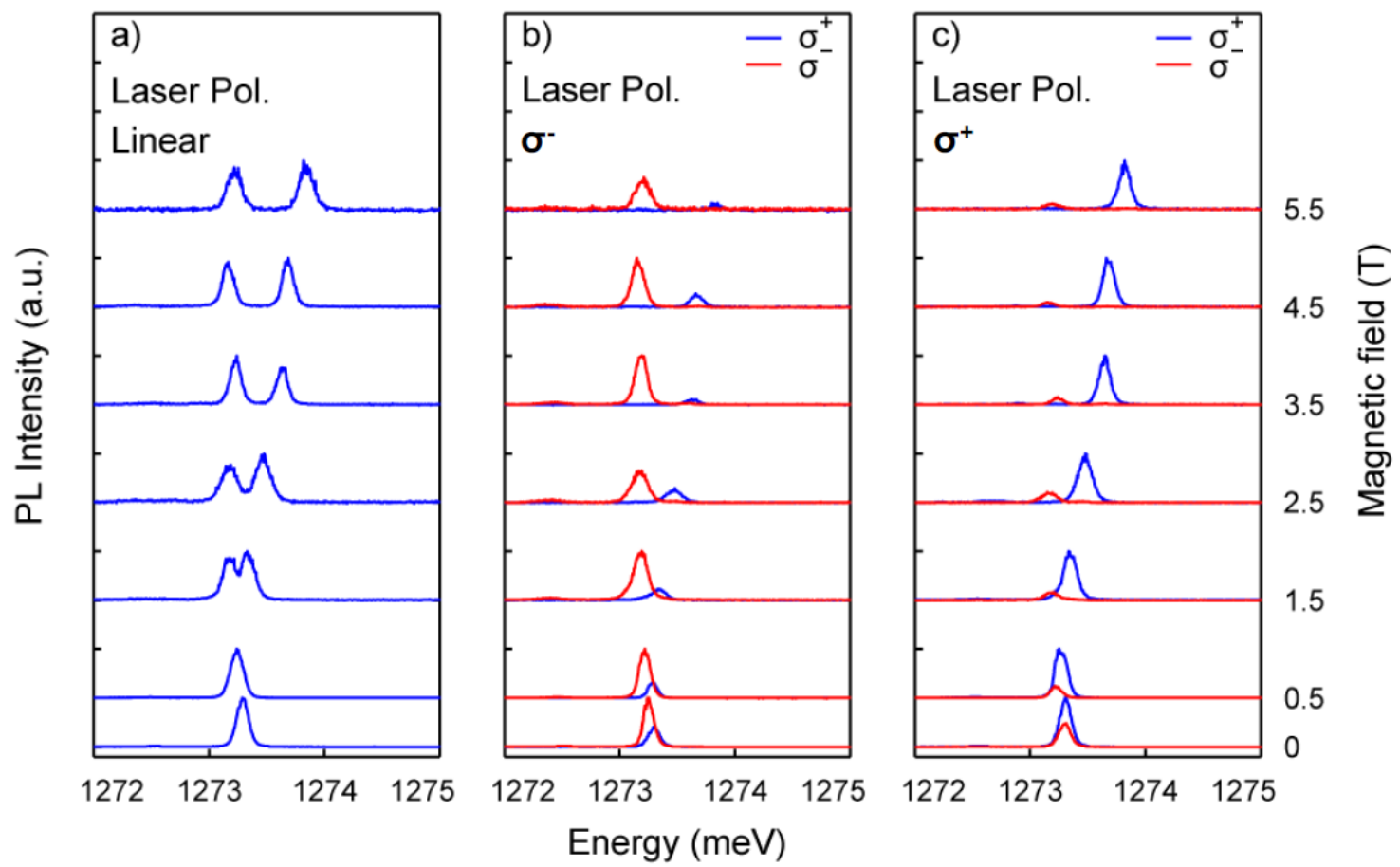

Figure 3. Polarization-selective excitation and detection of exciton spin states using quasi-resonant excitation. Both excitation and detection polarizations are varied. (a-c) PL for different magnetic fields. Excitation is linearly (a), left circularly (b), and right circularly (c) polarized, respectively. Emission analyzers are set to linear in (a), right (blue), or left (red) circular polarization in (b-c). Samples grown by the Bakkers group.

\subsection{Charge tunable device}

Nanowires offer the unique opportunity of merging optics at the single photon level with transport measurements at the single electron level. A heterostructure quantum dot in a nanowire can be electrically addressed very efficiently: all the current flowing through the nanowire will necessarily flow through the quantum dot. This enables devices that would efficiently convert single electrons into single photons. On the other hand a charge control in a nanowire quantum dot can enable quantum memories or can be used for entanglement between polarization degrees of a photon and spin of an electron. To this end, we have fabricated nanowires containing a quantum dot and realized a charge tunable device.

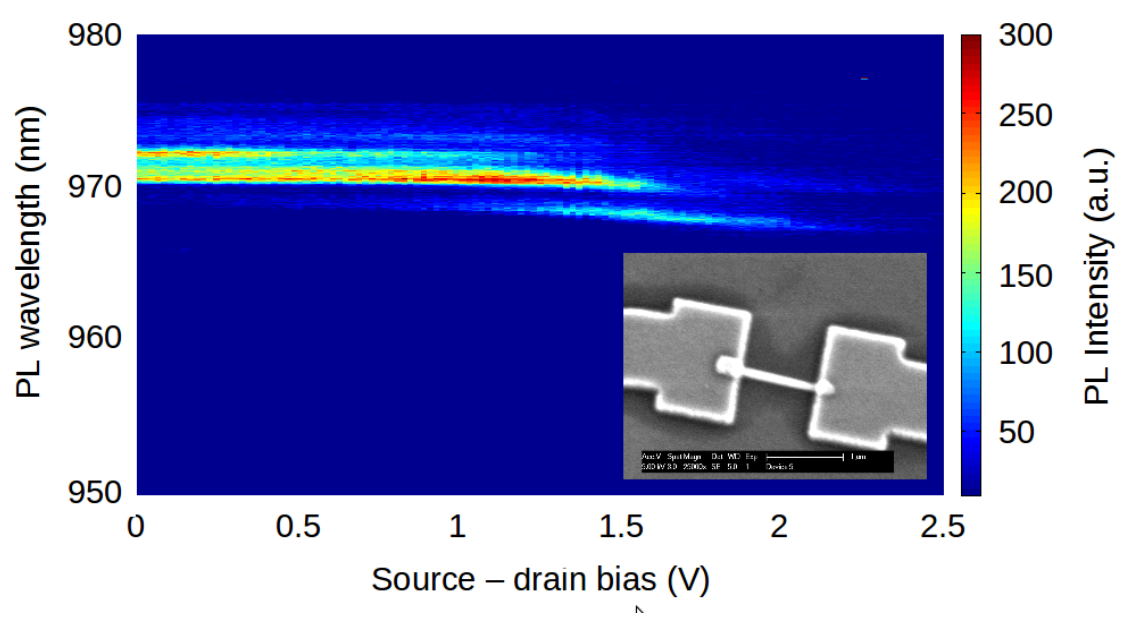

Figure 4. Contacted nanowire quantum dot device. PL as a function of source-drain bias. Different charge states are visible. The insert shows the SEM image of a typical device. Samples grown by the Bakkers group. 
The insert in Fig. 4 shows a contacted nanowire quantum dot used in our experiments. Using Schottky contacts and by applying a bias between them we change the electric field in the nanowire and bend the band profile. In analogy to selfassembled quantum $\operatorname{dots}^{22}$ the total charge in a nanowire quantum dot is changed that results in different PL lines. Our measurements under a non-resonant continuous wave laser excitation are shown in Fig. 4. We can clearly see that as we increase the bias the spectra change: the PL intensity of spectral lines at longer wavelength decreases and eventually quenches, while other spectral lines at shorter wavelength appear. At higher bias of $\sim 2.5$ Volts the PL is entirely quenched, meaning that the electric filed in the nanowire is stronger than the Coulomb interaction of the excitons and breaks them off. At this point a photocurrent flows through the nanowire. Three main spectral lines in the PL correspond to different charge states in the nanowire quantum dot and are controlled by the external electrical field applied to the device.

\section{CRYSTAL PHASE QUANTUM DOTS}

In semiconducting nanowires, both zincblende and wurtzite ${ }^{23}$ crystal structures can coexist ${ }^{24-26}$. The bandstructure difference between the two structures can lead to charge confinement ${ }^{27}$. Here we fabricate and study single quantum $\operatorname{dots}^{28}$ defined solely by crystal phase in a chemically homogeneous nanowire and observe single photon generation. More generally, our results show that this type of carrier confinement represents a novel degree of freedom in device design at the nanoscale.

In 1994 calculations predicted band offsets at WZ/ZB interfaces ${ }^{27}$. However, the first successful attempts to control the phase of a crystal structure during nanowire growth ${ }^{24,25}$ and the first optical studies ${ }^{29-32}$ were reported only recently. This progress practically enables engineering of the electronic structure of a single material. For example, a superlattice of twin planes can induce a direct bandgap in silicon ${ }^{33}$ that would have a strong impact in semiconductor technology; a WZ-ZB-WZ heterointerface can define a quantum well in a single material. Growing this structure in a nanowire will also confine charges in two other dimensions, forming a crystal phase quantum dot, which we demonstrate in this work.

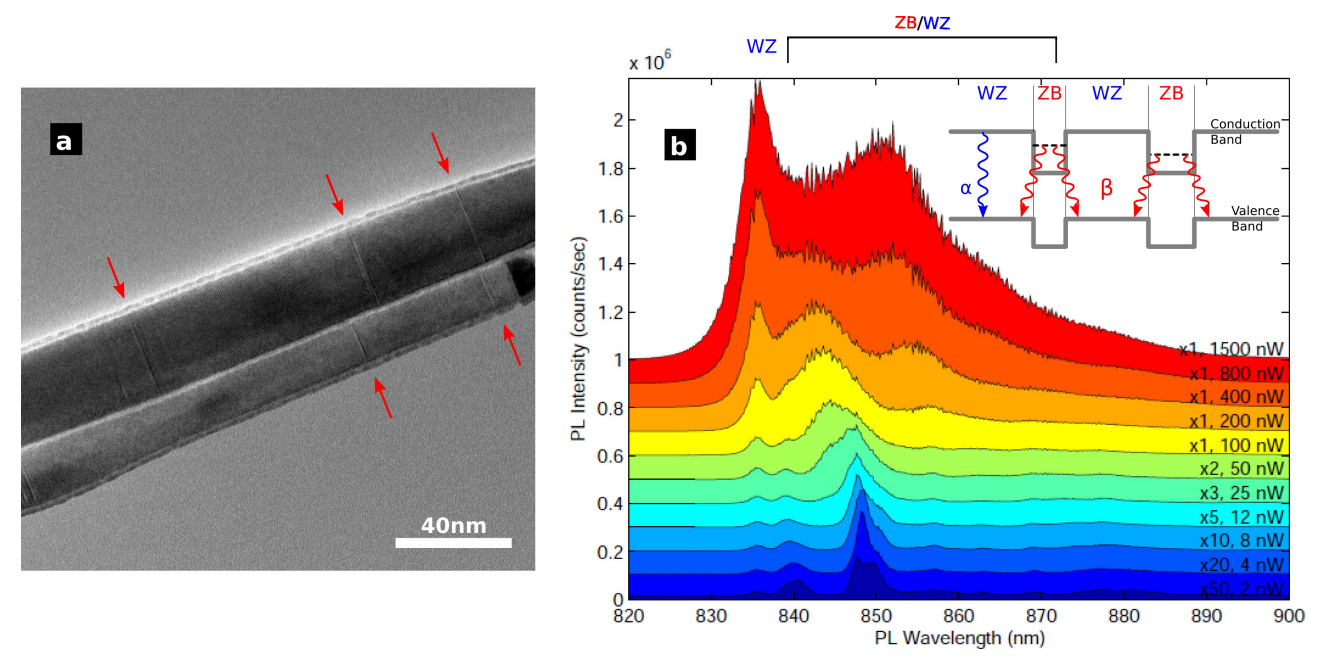

Figure 5. Crystal phase heterostructures. (a) Scanning transmission electron microscopy image of two InP nanowires. Few short ZB segments are visible and marked with red arrows. (b) Photoluminescence of a single InP nanowire under increasing excitation powers. Insert: schematics of the corresponding optical transitions from wurtzite and zincblende crystal structures respectively. $\alpha, \beta$ and $\gamma$ represent all possible optical transitions. MBE grown samples by the Harmand group.

The schematics of a crystal phase heterostructure is shown in the insert of Fig. 5b. The heterostructure is formed by growing indium phospide $(\mathrm{InP})$ nanowire in two different crystal phases: wurtzite and zincblende. Zincblende has a smaller bandgap and large negative band offset. Hence, the conduction and valence bands of zincblende segments are lower in energy compared to wurtzite parts. Short zincblende sections can confine electrons in the growth direction, and be barriers for holes. Two types of radiative recombinations are therefore possible. The first, labeled $\alpha$, is the direct recombination of electron-hole pairs in the wurtzite part of a nanowire, and therefore will result in a short lifetime. In the second transition, labeled $\beta$, electrons confined in zincblende segments will recombine radiatively with holes in adjacent wurtzite regions of a nanowire. This indirect electron-hole pair recombination will result in longer lifetimes, due to 
reduced overlap of electron and hole wavefunctions. In addition, electrons (and holes) can be confined in two other dimensions, perpendicular to the growth direction, with a small enough nanowire diameter. The three dimensional confinement will form a crystal phase quantum dot - it is formed from two different crystalline phases of the same material: wurtzite InP and zincblende InP, yielding quantized energy levels ${ }^{34}$. Their radiative recombination with holes in adjacent wurtzite parts of a nanowire will therefore be a source of singe photons ${ }^{9}$. The zincblende segments lengths set the confined electron energy levels. Accordingly, this will set energies and lifetimes of the emitted single photons. In Fig. 5a we show a transmission electron microscopy image of an InP nanowire where short zincblende segments are visible. Conversely to conventional heterostructures made of alternation of compounds, the crystal phase heterostructures have systematically an ideal interface with a step-like transition at the atomic scale. Photoluminescence of a single InP nanowire is shown in Fig. 5b. The spectral line at $835 \mathrm{~nm}$, labeled WZ, corresponds to electron-hole recombination of type $\alpha$ in the wurtzite part of the nanowire. Emission lines at longer wavelength, labeled ZB/WZ, correspond to electronhole recombinations of type $\beta$ in crystal phase quantum dots. With increasing excitation power the spectral lines broaden and blue shift due to energy states filling in quantum dots. The WZ line shows no spectral shift. This is expected since under the low excitation rates used here, the emission is predominantly governed by ground state electron-hole recombinations.

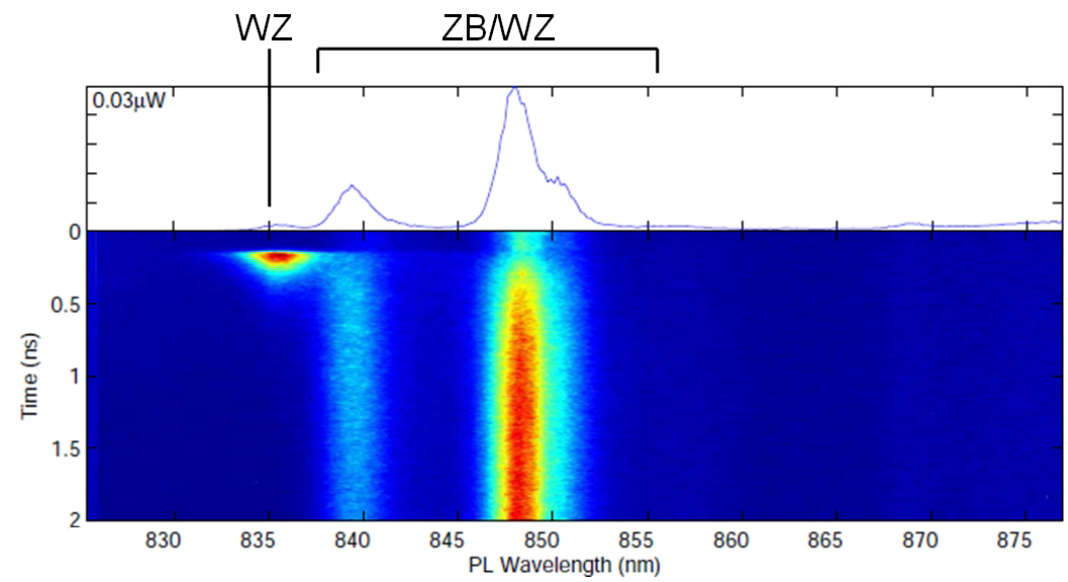

Figure 6. Time resolved photoluminescence of a single nanowire with crystal phase heterostructures. False-color plot represents time evolution of the PL after excitation by a laser pulse that arrives at time $0.2 \mathrm{~ns}$. The upper part represents the PL intensity integrated over the full time window of 2 ns. MBE grown samples by the Harmand group.

Time resolved photoluminescence measurements are represented in Fig. 6. We clearly observe a short lifetime, of the

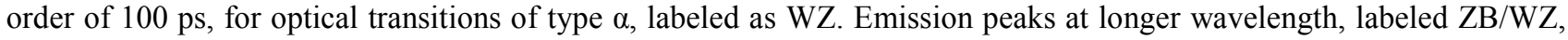
correspond to optical transitions of type $\beta$. Only a fraction of their decay traces are captured within a $2 \mathrm{~ns}$ time window, indicating much longer lifetimes. The data at times before $0.2 \mathrm{~ns}$ represent the part of the PL decay curve that was excited by the previous laser pulse $13 \mathrm{~ns}$ earlier. A significant signal measured at these times indicates that electrons and holes are trapped in short ZB and WZ sections for more than $13 \mathrm{~ns}$. Long lifetimes, measured in our experiments, lead to another important conclusion - a high purity of the sample. Defects or surface states in a nanowire that contribute to usually very fast non-radiative decay channels ${ }^{35}$, are negligible in these quantum dots.

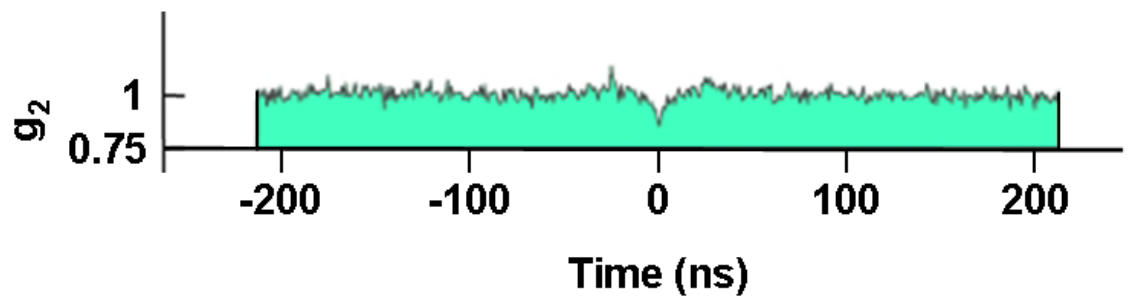

Figure 7. Second order auto-correlation measurement of photons emitted from a few crystal phase quantum dots under continuous wave laser excitation. MBE grown samples by the Harmand group. 
To demonstrate the quantum nature of crystal phase quantum dots we performed photon correlation measurements ${ }^{9,36}$ shown in Fig. 7. Here, PL signal from a line at $838 \mathrm{~nm}$ was filtered by a grating based filter, enabling $6 \mathrm{meV}$ resolution, and sent trough a fiber beamsplitter into two single photon detectors with $500 \mathrm{ps}$ temporal resolution. Missing correlation events at time 0 , antibunching, indicate that the emission originates from a few single photon emitters.

\section{CONCLUSIONS}

To conclude, we have demonstrated clean quantum dots in nanowires with narrow optical transitions. Specific exciton spin states are created and measured with properly polarized light. Electrical contacts enable the fabrication of optoelectronic devices where only one quantum dot is contacted, demonstrating a charge tunable device. In addition, we have shown formation of crystal phase quantum dots in a homogeneous InP nanowire. This new type of quantum dots independently expresses itself in four different types of experiments: transmission electron microscopy, photoluminescence spectroscopy, lifetime and photon correlation measurements. Natural charge separation allows for applications like quantum memories ${ }^{17}$ and solar cells ${ }^{37}$. These and other applications will also benefit from this clean, defect free system with sharp monolayer interfaces.

\section{REFERENCES}

1. H. Sakaki, Jpn. J. Appl. Phys. 19 (1980) L735-L738.

2. Y. Cui, C. Lieber, Science 291 (2001) 851-853.

3. E. Bakkers, J. van Dam, et al., Nature Mat. 3 (11) (2004) 769-773.

4. C. Fasth, A. Fuhrer, et al., Nano Lett. 5 (7) (2005) 1487-1490.

5. T. Sato, K. Hiruma, M. Shirai, K. Tominaga, K. Haraguchi, T. Katsuyama, T. Shimada, Appl. Phys. Lett. 66 (1995) 159.

6. T. Katsuyama, K. Ogawa, M. Koguchi, H. Kakibayashi, G.P. Morgan, Appl. Phys. Lett. 59 (1991) 431.

7. J.C. Johnson, H.J. Choi, et al., Nature Mat. 1 (2) (2002) 106-110.

8. M.T. Borgstrom, V. Zwiller, E. Muller, A. Imamoglu, Nano Lett. 5 (7) (2005) 1439-1443.

9. P. Michler, A. Kiraz, C. Becher, W.V. Schoenfeld, P.M. Petroff, Lidong Zhang, E. Hu, A. Imamoglu, Science 290 (2000) 2282.

10. E. Moreau, I. Robert, J.M. Gérard, I. Abram, L. Manin, V. Thierry-Mieg, Appl. Phys. Lett. 79 (2001) 2865.

11. V. Zwiller, H. Blom, P. Jonsson, N. Panev, S. Jeppesen, T. Tsegaye, E. Goobar, M.E. Pistol, L. Samuelson, G. Björk, Appl. Phys. Lett. 78 (2001) 2476.

12. N. Akopian, N.H. Lindner, et al., Phys. Rev. Lett. 96 (13) (2006).

13. R. Young, M. Stevenson, et al., New J. Phys. 8 (2) (2006) 29.

14. R. Hafenbrak, S.M. Ulrich, et al., New J. Phys. 9 (9) (2007) 315.

15. M. Tchernycheva, G. E. Cirlin, G. Patriarche, L. Travers, V. Zwiller, U. Perinetti, J.-C. Harmand, Nano Lett. 2007, 7, 1500-1504.

16. F. Glas, J.-C. Harmand, G. Patriarche, Phys. Rev. Lett. 2007, 99, 146101.

17. M. Kroutvar, Y. Ducommun, D. Heiss, M. Bichler, D. Schuh, G. Abstreiter, J. J. Finley, Nature (London) 2004, 432, $81-84$.

18. M. H. M. van Weert, N. Akopian, U. Perinetti, M. P. van Kouwen, R. E. Algra, M. A. Verheijen, E. P. A. M. Bakkers, L. P. Kouwenhoven, V. Zwiller, Nano Lett. 2009, 9, 1989-1993.

19. A. Greilich, A. Shabaev, D. R. Yakovlev, Al. L. Efros, I. A. Yugova, D. Reuter, A. D. Wieck, M. Bayer, Science 2007, 317, 1896-1899.

20. M. Senes, B. Urbaszek, X. Marie, T. Amand, J. Tribollet, F. Bernardot, C. Testelin, M. Chamarro, J.-M. Gerard, Phys. ReV. B 2005, 71, 115334.

21. M. Paillard, X. Marie, P. Renucci, T. Amand, A. Jbeli, J.-M. Gerard, Phys. ReV. Lett. 2001, 86, 1634-1637.

22. E. Poem, J. Shemesh, I. Marderfeld, D. Galushko, N. Akopian, D. Gershoni, B. D. Gerardot, A. Badolato, P. M. Petroff, Phys. Rev. B 76, 235304 (2007).

23. P. Y. Yu, M. Cardona, Fundamentals of Semiconductors: Physics and Materials Properties. (Springer, Berlin, 2005).

24. R. E. Algra, M. A. Verheijen, M. T. Borgstrom, L. F. Feiner, G. Immink, W. J. P. van Enckevort, E. Vlieg, E. P. A. M. Bakkers, Nature 2008, 456, 369-372.

25. P. Caroff, K. A. Dick, J. Johansson, M. E. Messing, K. Deppert, L. Samuelson, Nature Nanotech. 2009, 4, 50-55. 
26. M. Koguchi, H. Kakibayashi, M. Yazawa, K. Hiruma, T. Katsuyama, Jpn. J. Appl. Phys. 1992, 31, 2061-2065.

27. M. Murayama, T. Nakayama, Phys. Rev. B 1994, 49, 4710-4724.

28. X. Duan, Y. Huang, Y. Cui, J. Wang, C. M. Lieber, Nature 2001, 409, 66-69.

29. B. W. Jacobs, V. M. Ayres, R. E. Stallcup, A. Hartman, M. A. Tupta, A. D. Baczewski, M. A. Crimp, J. B. Halpern, M. He, H. C. Shaw, Nano Lett. 2007, 7, 1435-1438.

30. J. Bao, D. C. Bell, F. Capasso, J. B. Wagner, T. Martensson, J. Tradgardh, L. Samuelson, Nano Lett. 2008, 8, 836841.

31. K. Pemasiri, M. Montazeri, R. Gass, L. M. Smith, H. E. Jackson, J. Yarrison-Rice, S. Paiman, Q. Gao. H. H. Tan, C. Jagadish, X. Zhang, J. Zou., Nano Lett. 2009, 9, 648-654.

32. T. B. Hoang, L. V. Titova, H. E. Jackson, L. M. Smith, J. M. Yarrison-Rice, J. L. Lensch, L. J. Lauhon, Appl. Phys. Lett. 2009, 94, 133105.

33. Z. Ikonic, G. P. Srivastava, J. C. Inkson, Phys. Rev. B 1995, 52, 14078-14085.

34. E. Dekel, D. Gershoni, E. Ehrenfreund, D. Spektor, J. M. Garcia, P. M. Petroff, Phys. Rev. Lett. 1998, 80, 49914994.

35. M. Mattila, T. Hakkarainen, H. Lipsanen, H. Jiang, E. I. Kauppinen, Appl. Phys. Lett. 2007, 90, 033101.

36. R. Hanbury-Brown, R. Q. Twiss, Nature 1956, 177, 27-29.

37. B. Tian, X. Zheng, T. J. Kempa, Y. Fang, N. Yu, G. Yu, J. Huang, C. M. Lieber, Nature 2007, 449, 885-889. 